\title{
Particle Acceleration through Multiple Conversions from Charged into Neutral State and Back
}

\author{
E.V. Derishev ${ }^{1,2}$, F.A. Aharonian ${ }^{1}$, V.V. Kocharovsky ${ }^{2,3}$, Vl.V. Kocharovsky ${ }^{2}$ \\ ${ }^{1}$ MPI für Kernphysik, Saupfercheckweg 1, D-69117 Heidelberg, Germany \\ ${ }^{2}$ Institute of Applied Physics RAS, 46 Ulyanov st., 603950 Nizhny Novgorod, Russia \\ ${ }^{3}$ Dept. of Physics, Texas A\&M University, College Station, TX 7r843-4242
}

\begin{abstract}
We propose a new means for quick and efficient acceleration of protons and/or electrons in relativistic bulk flows. The maximum attainable particle energies are limited either by radiative losses or by the condition of confinement in the magnetic field. The new mechanism takes advantage of conversion of particles from the charged state (protons or electrons/positrons) into neutral state (neutrons or photons) and back. In most cases, the conversion is photon-induced and requires presence of intense radiation fields, but under special circumstances the converter acceleration mechanism may operate via other charge-changing reactions, for example, inelastic nucleon-nucleon collisions. Like in the traditional, "stochastic" (or diffusive) acceleration models, the acceleration cycle in the proposed scenario consists of escape of particles from the relativistic flow followed by their return back after deflection from the ambient magnetic field. The difference is that the charge-changing reactions, which occur during the cycle, allow accelerated particles to increase their energies in each cycle by a factor much larger than 2 and usually roughly equal to the bulk Lorentz factor squared. The emerging spectra of accelerated particles can be very hard and their maximum energy in some cases is larger than in the standard mechanism. This significantly reduces the required energy budget of the sources of the highest-energy particles observed in cosmic rays. acceleration mechanism has a distinctive feature - it unavoidably creates neutral beams, consisting of photons, neutrinos or neutrons, whose beam pattern may be much broader than the inverse Lorentz factor of the relativistic flow. Also, the new mechanism may serve as an efficient means of transferring the energy of bulk motion to gamma-radiation and, if the accelerated particles are nucleons, inevitably produces high-energy neutrinos at relative efficiency approaching $\gtrsim 50 \%$.
\end{abstract}

PACS numbers: $98.70 . \mathrm{Sa}, 98.62 . \mathrm{Js}, 98.70 . \mathrm{Rz}$

\section{INTRODUCTION}

The cosmic rays (CRs) have been studied for a long time (see, e.g., [1] for a review). There are many models of their (likely non-uniform) sources, but explaining the origin of the highest-energy particles $\left(\gtrsim 10^{20} \mathrm{eV}\right)$ observed in CRs is still a challenging problem 2]. In general, two scenarios have been proposed for the particle acceleration in astrophysical environments. One is the acceleration by electric field in geometries, where this field is not perpendicular to magnetic field-lines, for example, in the vicinity of magnetized rotating neutron stars [3]. Very hard particle spectra may emerge in this way, but, because of the curvature losses inherent to such geometries, the upper limit to proton energy appears to be below $10^{20} \mathrm{eV}$.

Another class of scenarios assumes gradual, "stochastic" (or diffusive) acceleration of charged particles through multiple reflections from inhomogeneities of magnetic field in the environments where large velocity gradients are present (see [4] for a review). According to the generally accepted view (see, e.g., $[4,5,6]$ ) this mechanism works equally well for shocks and shear flows, and the emerging spectra of accelerated particles are such, that only a small fraction of the total energy budget is contained in the most energetic particles.

Furthermore, there is a serious obstacle in achieving high energies of the accelerated particles - their diffusive escape. In the environments, where bulk velocities are sub-relativistic $(v \ll c)$, it takes many reflections to increase the energy of accelerated particle twofold. In this case, the mechanism can work only in the largest objects in the Universe, like galaxy clusters or radio lobes and knots in active galactic nuclei (AGNs), and only under optimistic assumption about the diffusion coefficient for particles in magnetic field. For example, Bohm diffusion leads to particle escape on the timescale $R_{0}^{2} / r_{g} c$, where $R_{0}$ is the accelerator's size and $r_{g}$ the particle's gyroradius, while the acceleration occurs on the timescale $\sim(c / v)^{2} r_{g} / c$. The maximum attainable energy is defined by equating these timescales: $r_{g}=(v / c) R_{0} \ll R_{0}$. It is of the order of $10^{19} \mathrm{eV}$ in the most favorable cases.

Ultrarelativistic shocks and shear flows are more promising, since the parameter $v / c \rightarrow 1$. Moreover, the energy gain per cycle, i.e., at each reflection from the shock or from the shear flow boundary, can approach the factor of $\sim \Gamma^{2}$, where $\Gamma$ is the bulk Lorentz factor (measured in the upstream fluid frame in the case of shock). Such a scenario was suggested, e.g., as the dominant acceleration mechanism in gamma-ray bursts (GRBs) 7, 8, 9]. However, in the standard acceleration theory, the energy gain of $\sim \Gamma^{2}$ occurs only at the first cycle, while the subsequent ones result in energy gain of $\sim 2$ each [10, 11].

The reason is that the shock catches up to the reflected particle (or the particle crosses the boundary of the shear 


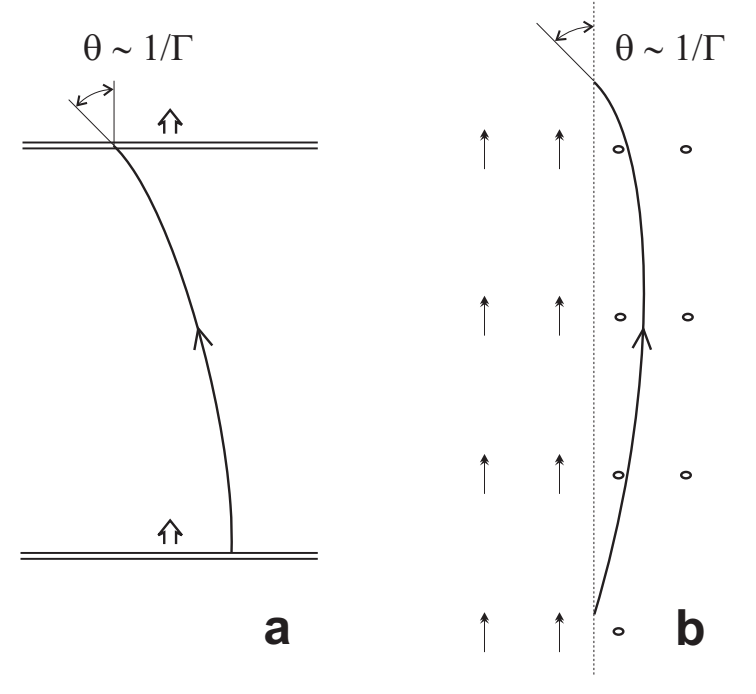

FIG. 1: The acceleration cycle in the standard mechanism for a shock (a) and for a shear flow (b). The thick solid line shows the particle's trajectory. The magnetic field is perpendicular to the picture plane. The locations of the shock at the moments of particle escape from the shock and subsequent catch-up are shown as double lines. The shear flow boundary is shown by thin dotted line.

flow) when its trajectory makes an angle of $\simeq 2 / \Gamma$ to the shock normal (or bulk velocity vector), while keeping the $\Gamma^{2}$ energy gain would require isotropisation.

The smallness of this angle is apparent for the case of shear flow (see Fig. 1b). For shocks it is a consequence of relativistic motion (Fig. 1a). Indeed, the component of particle velocity along the shock normal becomes smaller than the velocity of the shock itself as soon as the angle between the particle's momentum and the shock normal grows larger than $1 / \Gamma$. There is no apparent way to isotropize reflected particles unless a special structure in the magnetic field ahead of the shock is introduced, e.g., a counter-propagating shock.

However, a means to circumvent these limitations exists [12] - it can be done by switching particle's charge on and off at right times. Paradoxically, interactions with photons, which have been always treated as dissipative processs leading to degradation of particle energy, in fact play positive role: they allow (through the chargechanging particle conversion) to keep the $\Gamma^{2}$ energy gain up to the largest particle energies. There are also other types of conversion reactions, which we briefly discuss in the following section. One of the suggested below charge-changing schemes (electron-photon-electron reaction chain in GRB shocks) is independently considered in [13].

In order to outline the general picture, we intentionally skip some details, which are not essential for the proposed acceleration mechanism, but may change its quantitative characteristics. In particular, we assume that the magnetic field is either chaotic (turbulent) or uniform with field lines perpendicular to both the momentum of accelerated particle and the velocity of the flow, and treat shocks and shear flows as one-dimensional discontinuities.

We use the convention $F_{\nu} \propto \nu^{q}$ to define the spectral index $q$, where $F_{\nu}$ is the energy flux per unit frequency interval.

\section{THE CONVERTER ACCELERATION MECHANISM}

Two basic types of photon-induced conversion involve nucleons or electrons/positrons. Both cycles consist of two reactions:

$$
p+\gamma \rightarrow n+\pi^{+} \quad \text { and } \quad n+\gamma \rightarrow p+\pi^{-}
$$

for proton cycle,

$$
e^{ \pm}+\gamma \rightarrow e^{ \pm}+\gamma^{\prime} \quad \text { and } \quad \gamma^{\prime}+\gamma \rightarrow e^{+}+e^{-}
$$

for electron cycle. Here $p, n, \pi^{ \pm}$, and $e^{ \pm}$denote proton, neutron, charged pions (positive and negative), positron and electron, respectively; $\gamma$ is a low-energy background photon and $\gamma^{\prime}$ the high-energy comptonized photon.

The second of reactions from the electron cycle (2) has a kinematic threshold $\Delta_{e}=2 m_{e} c^{2}$ in the center-ofmomentum frame, where $m_{e}$ is the electron mass. Effectively, the first reaction also has the threshold $\simeq \Delta_{e}$, since at lower energies of incident photons the efficiency of energy transfer to the comptonized photon becomes much less than unity. The reactions proceed differently depending on the background photon spectrum. Soft spectrum blocks the electron cycle as the fraction of energy transferred to a comptonized photon is too small and there are few target photons sufficiently energetic for the second reaction of the electron cycle. An example of soft spectrum could be a power-law with spectral index $q<-1$ or a narrow-band spectrum, like black-body or line emission, with typical photon energy $\bar{\varepsilon} \ll m_{e}^{2} c^{4} / \varepsilon_{e}$, where $\varepsilon_{e}$ is the electron energy. For intermediate spectra (e.g., power-laws with indices $-1<q<1$ or narrowband spectrum with $\bar{\varepsilon} \sim m_{e}^{2} c^{4} / \varepsilon_{e}$ ), the comptonized photon takes about $1 / 2$ of the electron (positron) energy, and in the consequent pair production event this energy is divided nearly in equal parts between the daughter electron and positron. The cross-section in both processes is $\sigma_{e, \gamma} \sim 10^{-25} \mathrm{~cm}^{2}$. For hard spectra $(q>1$ or $\left.\bar{\varepsilon} \gg m_{e}^{2} c^{4} / \varepsilon_{e}\right)$, these reactions proceed in the deep Klein-Nishina regime, i.e., the comptonization and pairproduction cross-sections decrease inversely proportional to the square of the center-of-momentum energy (their ratio is 1:2) and almost all the energy of interacting particles is transferred to one of the daughter particles. In effect, the energy losses for the combined electron/photon particle become very gradual. This case is the closest to the pure conversion (the probability of charge change $p_{c}=1$ ) without accompanying energy losses provided the synchrotron emission is negligible. 
The reactions from the proton cycle (11) (see, e.g., 14]) have kinematic threshold of $\left(m_{\pi} c^{2}+m_{\pi}^{2} c^{2} / 2 m_{N}\right) \simeq$ $150 \mathrm{MeV}$ in the nucleon rest frame $\left(m_{\pi} \simeq 140 \mathrm{MeV} / c^{2}\right.$ is the charged pion mass and $m_{N} \simeq 940 \mathrm{MeV} / c^{2}$ the nucleon mass). Side by side with the reactions (1) proceed other photopionic reactions with formation of neutral pion, which preserve nucleon's charge. They have roughly the same cross-section and should be considered as a background energy losses. The total photopionic cross-section rapidly increases with energy of incident photon and reaches maximum of $\sigma_{\pi} \simeq 6 \times 10^{-28} \mathrm{~cm}^{2}$ at $\Delta_{p} \simeq 340 \mathrm{MeV}$, which corresponds to formation of $\Delta$ resonance and should be considered as effective threshold. Well above the resonance energy, the cross-section decreases and levels off at $\simeq 10^{-28} \mathrm{~cm}^{2}$. The probability of charge change in a photopionic reaction is $p_{c} \simeq 1 / 3$ at the resonance and $p_{c} \simeq 1 / 2$ at the plateau. The inelasticity is $\simeq 0.2$ and about 0.5 , respectively.

A competing photon-induced reaction is the process of creation of an electron-positron pair by a photon interacting with the electric field of a proton $p+\gamma \rightarrow p+e^{-}+e^{+}$, which has the cross-section $\simeq 5 \times 10^{-27} \mathrm{~cm}^{2}$ and inelasticity $\simeq 10^{-3}$. With the decrease of spectral index $q$ of target photon field, this process becomes an increasingly important energy loss channel, and at $q \simeq-1.55$ the difference with the photopionic processes in the inelasticity and cross-section is exactly balanced by larger number of target photons (thanks to lower threshold). Anyway, the $p+\gamma \rightarrow p+e^{-}+e^{+}$process can be neglected for spectra with $q \gtrsim-1.5$.

In dense environments and at relatively low nucleon energies, i.e., in the case where there are few target photons, the proton cycle proceeds through inelastic nucleonnucleon collisions, for example,

$$
p+p \rightarrow n+p+\pi^{+} \quad \text { and } \quad n+p \rightarrow p+p+\pi^{-} .
$$

The kinematic threshold for these reactions is $m_{\pi} c^{2}$ in the center-of-momentum frame, and the cross-section at energies $\gg m_{\pi} c^{2}$ is $\simeq 3 \times 10^{-26} \mathrm{~cm}^{2}$. The acceleration via inelastic nucleon-nucleon collisions could be important in GRB internal shocks, where the required column density of $\sim 10 \mathrm{~g} / \mathrm{cm}^{2}$ is achieved [15].

In both proton and electron cycles, one can consider an accelerated nucleon or electron/positron as a particle, which has both charged and neutral states. The acceleration cycle consists of three steps (see Fig. 2). First, a charged particle in relativistic flow is converted into neutral state (point 1). Then, experiencing no influence from the magnetic field, it freely leaves the flow and propagates into ambient medium much further than if it were charged. Second, a transition from neutral to charged state occurs (point 2), which may be the spontaneous neutron decay.

At this moment, the particles in the laboratory frame preserve beaming with the opening angle of $\sim 1 / \Gamma$, which they had in the neutral state. The initial handicap allows particles to be deflected by an angle $\theta \gg 2 / \Gamma$ before the encounter with the relativistic flow. The angular
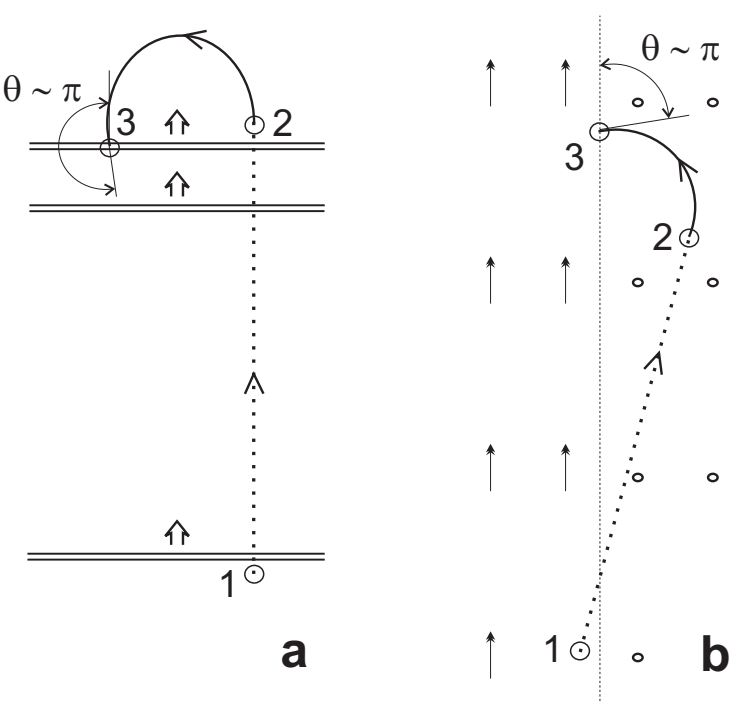

FIG. 2: The acceleration cycle in the converter mechanism for a shock (a) and for a shear flow (b). The particle's trajectory is shown by thick dotted line (neutral state) and thick solid line (charged state). The magnetic field is perpendicular to the picture plane. Numbered are the moments of particle conversion into neutral state, transition from neutral to charged state, and subsequent return to the flow. The locations of the shock at the corresponding moments are shown by double lines. The shear flow boundary is shown by thin dotted line.

spreading of the particle beam in the laboratory frame means energy gain in the flow comoving frame, which is much larger than 2 and amounts to $\Gamma^{2}$ in the case of full isotropisation. At the third step, the particles return to the flow (point 3) and their isotropisation in the comoving frame translates into the resulting energy gain in the laboratory frame.

The main parameter, characterizing the efficiency of the converter mechanism, is the optical depth for interactions of accelerated particles (protons/neutrons or electrons/photons) $\tau=\sigma n D$, where $n$ is the number density of target particles (photons or nucleons) and $D$ the accelerator's size, both measured in the comoving frame, $\sigma$ the relevant cross-section.

The optical depth is geometry-dependent. In the case of a continuous outflow or a shock, produced by a central engine and subtending an angle $>1 / \Gamma$, one has for photon-induced reactions

$$
\tau \simeq \frac{\sigma L\left(\varepsilon_{*}\right) \Theta^{2}}{4 \pi R c \varepsilon_{*}} .
$$

Here $L$ is the apparent luminosity per logarithmic frequency interval at photon energy $\varepsilon_{*}=2 m c^{2} \Delta /\left(\varepsilon \Theta^{2}\right)$, where the interaction with target photons is the most efficient, $\varepsilon$ the energy of accelerated particle, $R$ the distance from the central engine, $\Delta$ and $m$ are the threshold and the mass of the particle for one of the possible cycles.

The beaming angle of target radiation field is $\Theta \sim 1 / \Gamma$ in the case where it is produced within the jet or by the shocked gas, and $\Theta \sim 1$ for the emission from broad-line 
regions in AGNs and the radiation scattered in the interstellar medium around GRBs. Intermediate cases, where $1 / \Gamma<\Theta<1$, are also possible. They include, for example, the radiation from inner parts of accretion discs in AGNs or the emission which accompanies the acceleration process (as explained in Sect. V). If the source of target radiation is transient, like a GRB, with duration less than $R \Theta^{2} / c$, then its luminosity should be replaced by $E c /\left(R \Theta^{2}\right)$, where $E=\int L d t$.

The most favorable for the converter mechanism conditions exist in AGNs and GRBs, where both ultrarelativistic flows and intense radiation fields are present (see [16] and [17 for reviews). Other objects with relativistic outflows, e.g., stellar-mass microquasars, may also be able to accelerate particles via the converter mechanism. The only two prerequisites are sufficiently high conversion probability (which we specify below) and bulk Lorentz factor $\Gamma \gg 2$ in order to compensate the energy losses caused by conversion.

Because of relatively large cross-section, the optical depth for photon-induced reactions is not a bottleneck for the electron cycle, but it could be a limiting factor for the proton cycle. Let us estimate the optical depth for photopionic reactions in three cases. For AGN inner jets we obtain (taking into account only comoving photon fields with $\Theta=1 / \Gamma)$

$$
\tau \simeq 10^{-1}\left(\frac{L\left(\varepsilon_{*}\right)}{10^{45} \mathrm{erg} / \mathrm{s}}\right)\left(\frac{\varepsilon}{10^{18} \mathrm{eV}}\right)\left(\frac{10}{\Gamma}\right)^{4}\left(\frac{10^{15} \mathrm{~cm}}{R}\right),
$$

where the apparent luminosity per logarithmic frequency interval $L\left(\varepsilon_{*}\right)$ depends on $\varepsilon$. The radiation from AGN broad-line regions creates the optical depth

$$
\tau \sim 5 \times 10^{-2}\left(\frac{L}{10^{44} \mathrm{erg} / \mathrm{s}}\right)\left(\frac{10 \mathrm{eV}}{\bar{\varepsilon}}\right)\left(\frac{10^{17} \mathrm{~cm}}{R}\right),
$$

which does not depend on the particle's energy: for all particles with energy $\varepsilon>2 m c^{2} \Delta / \bar{\varepsilon} \simeq 5 \times 10^{16} \mathrm{eV}$ the number of target photons is essentially constant because of their relatively narrow spectral distribution. In GRBs, the optical depth due to comoving photons is

$\tau \simeq 3 \times 10^{-3}\left(\frac{E\left(\varepsilon_{*}\right)}{10^{52} \mathrm{erg}}\right)\left(\frac{\varepsilon}{10^{16} \mathrm{eV}}\right)\left(\frac{100}{\Gamma}\right)^{2}\left(\frac{10^{16} \mathrm{~cm}}{R}\right)^{2}$.

So, the conversion probability $p_{\mathrm{cn}}=\left[1-\exp \left(-p_{c} \tau\right)\right]$ in the proton cycle is usually, though not always, much smaller than unity.

It should be noted, that effectively the probability of conversion of a neutron into a proton always exceeds

$$
p_{\mathrm{cn}}^{(\min )}=\frac{R m_{N} c}{t_{n} \varepsilon} \simeq 3 \times 10^{-2}\left(\frac{10^{15} \mathrm{eV}}{\varepsilon}\right)\left(\frac{R}{10^{18} \mathrm{~cm}}\right)
$$

because of the neutron decay. Here $t_{n} \simeq 900 \mathrm{~s}$ is the lifetime of free neutron. The spontaneous decay of free neutrons is important at small energies, especially during the first acceleration cycle, while at large energies the photon-induced conversion is more efficient.

The expressions (5), (6) and (7) cover all physically different situations. For example, one may use Eq. (6) to estimate the optical depth in microquasars, where the target photons are produced by a hot corona having the size $R \sim 10^{8} \mathrm{~cm}$ and the luminosity $L \sim 10^{37} \mathrm{erg} / \mathrm{s}$. The result is $\tau \sim 0.1$ at target-photon energy of $\sim 1 \mathrm{keV}$, so that the proton cycle may operate in microquasars at energies $\gtrsim 3 \times 10^{14} \mathrm{eV}$. It is possible, therefore, that some contribution to the galactic CRs around the knee comes from microquasars. In any case, we see no problem in realization of the electron cycle in such objects.

\section{ENERGY GAIN}

Assuming that momenta of particles are isotropized in the comoving frame upon their encounter with the relativistic flow, one gets the average energy gain per cycle

$$
g \simeq \frac{(\Gamma \theta)^{2}}{2}
$$

where $\theta$ is the angle between the particle's momentum and the flow's velocity (deflection angle) at the moment of encounter. If the deflection angle is small, $\theta \ll 1$, then it grows linearly with distance $\ell$ (travelled by the particle in the charged state)

$$
\theta=\theta_{0}+\ell / r_{g}
$$

in uniform magnetic field, while in chaotic field it behaves as

$$
\langle\theta\rangle=\sqrt{\theta_{0}^{2}+\ell \ell_{c} / r_{g}^{2}}
$$

on average. Here $\theta_{0}$ is the initial deflection angle, measured at the time of conversion from neutral to charged state, $\ell_{c}<\ell$ the turbulence scale of the magnetic field, and $r_{g}$ the gyroradius of the particle, calculated as if the field were uniform. Note, that the turbulence scale is implicitly defined by Eq. (11) and, therefore, may depend on the particle's energy.

In the case of acceleration at the shock front, the shock catches up to the particle when the displacement of the particle along the shock normal, after it crossed the shock, becomes equal to the distance traveled by the shock front, i.e.,

$$
\frac{\sqrt{\Gamma^{2}-1}}{\Gamma}\left(\ell_{0}+\ell\right)=\ell_{0} \cos \theta_{0}+\int_{0}^{\ell} \cos \theta d \ell^{\prime},
$$

where $\ell_{0}$ is the distance travelled by the particle in the neutral state after it left the relativistic flow, and we neglected the difference between the particle's velocity and the velocity of light. 
In the uniform magnetic field, the deflection angle is

$$
\theta \simeq\left\{\frac{3 \ell_{0}}{r_{g} \Gamma^{2}}\left(1-\Gamma^{2} \theta_{0}^{2}\right)\right\}^{1 / 3} \sim\left(\frac{3 \ell_{0}}{r_{g} \Gamma^{2}}\right)^{1 / 3}
$$

provided $r_{g} / \Gamma \ll \ell_{0} \ll r_{g} \Gamma^{2}$. For smaller initial displacement, one arrives to the result of standard theory $\theta \simeq 1 / \Gamma$, whereas for larger displacement the deflection angle is $\theta \sim 1$. In the rightmost part of Eq. (13) we assume that $\left(1-\Gamma^{2} \theta_{0}^{2}\right) \sim 1$ to simplify the algebra. This corresponds to ignoring particles, which escape propagating (in the comoving frame) nearly parallel to the shock plane. They are neither numerous nor energetically important.

If one substitutes $\ell_{0}$ by $R$ (or by $\Gamma D$ in the case of a small blob with the size $D<R / \Gamma$, ejected by a central engine), then the applicability limits give two critical energies (both measured at the end of acceleration cycle point 3 in Fig. 2):

$$
\varepsilon_{1}=\Gamma e B R \quad \text { and } \quad \varepsilon_{2}=e B R
$$

where $e$ is the charge of the accelerated particle and $B$ the magnetic field strength. The acceleration proceeds with the maximum energy gain of $\sim \Gamma^{2}$ up to the energy $\varepsilon_{2}$, whereas above the energy $\varepsilon_{1}$ there is no advantage over the standard mechanism in the energy gain. At the same time, $\varepsilon_{1}$ is equal to the maximum energy, achievable in the standard mechanism.

In the chaotic magnetic field

$$
\langle\theta\rangle \simeq\left\{\frac{2 \ell_{c} \ell_{0}}{r_{g}^{2} \Gamma^{2}}\left(1-\Gamma^{2} \theta_{0}^{2}\right)\right\}^{1 / 4} \sim\left(\frac{2 \ell_{c} \ell_{0}}{r_{g}^{2} \Gamma^{2}}\right)^{1 / 4}
$$

for $r_{g}^{2} / \Gamma^{2} \ll \ell_{c} \ell_{0} \ll \Gamma^{2} r_{g}^{2}$, and the critical energies are equal,

$$
\varepsilon_{1}=\varepsilon_{2}=\Gamma e B \sqrt{R \ell_{c}} .
$$

In the case of shear flow, the catch-up condition reads

$$
\ell_{0} \sin \theta_{0}=\int_{0}^{\ell} \sin \theta d \ell^{\prime}
$$

The deflection angle is

$$
\theta \simeq\left(2 \frac{\ell_{0} \theta_{0}}{r_{g}}\right)^{1 / 2} \sim\left(\frac{2 \ell_{0}}{r_{g} \Gamma}\right)^{1 / 2}
$$

for uniform field $\left(r_{g} / \Gamma \ll \ell_{0} \ll r_{g} \Gamma\right)$ and

$$
\langle\theta\rangle \simeq\left(\frac{3}{2} \frac{\ell_{0} \ell_{c} \theta_{0}}{r_{g}^{2}}\right)^{1 / 3} \sim\left(\frac{3}{2} \frac{\ell_{0} \ell_{c}}{r_{g}^{2} \Gamma}\right)^{1 / 3}
$$

for chaotic field $\left(r_{g}^{2} / \Gamma^{2} \ll \ell_{0} \ell_{c} \ll r_{g}^{2} \Gamma\right)$.

There is a subtlety in realization of the converter mechanism if acceleration takes place at a shear flow boundary. If the charged particle reappears at a distance greater than $r_{g}$ (uniform magnetic field) or $r_{g}^{2} / \ell_{c}$ (chaotic field) from the flow boundary, then it should drift or diffuse back. It takes a time of the order of $t_{d}=R^{2} /\left(\Gamma r_{g} c\right)$, in the least favourable case of quasiuniform magnetic field with characteristic spatial scale of $\sim R$, or $t_{d}=R^{2} \ell_{c} /\left(\Gamma^{2} r_{g}^{2} c\right)$, in chaotic magnetic field. In both cases we assumed $\ell_{0} \sin \theta_{0}=R / \Gamma$. The solution of diffusion problem with a sink (the shear flow) in the half-space shows, that all particles eventually return to the flow, provided it persists for sufficiently long time. In reality, some of them are lost because the shear flow does not occupy the entire half-space, but the losses are negligible if the spatial extent of the shear flow boundary is much larger than $\ell_{0} \sin \theta_{0}$. This condition, for example, is satisfied for a conical jet with the opening angle $\gg 1 / \Gamma$.

The synchrotron losses during the time $t_{d}$ might be a more serious problem. One can neglect them if the energy loss rate $\dot{\varepsilon}=4 / 9\left(e^{2} / m c^{2}\right)^{2} B^{2}\left(\varepsilon / m c^{2}\right)^{2} c$ multiplied by $t_{d}$ is less than the energy of accelerated particle. In the case of quasi-uniform magnetic field, this condition turns out to be energy-independent and, after simple algebra, one gets

$$
R \gg \frac{4}{9} \frac{1}{\Gamma^{4}} \frac{e^{2}}{m c^{2}}\left(\frac{\varepsilon_{2}}{m c^{2}}\right)^{3}, \quad \text { i.e., } \quad R \gg \frac{R_{\mathrm{opt}}}{\Gamma^{2}} .
$$

Here $R_{\text {opt }}$ is the optimal size of an electromagnetic accelerator [2], which is defined to minimize the amount of energy contained in the electric and magnetic fields. Let us recall, however, that the optimal size is originally defined for the accelerator itself (in this case - for the interior of the shear flow), while Eq. (20) has to do with the external magnetic field.

In a turbulent magnetic field, the synchrotron losses are the most significant for the least energetic particles, i.e., those having $r_{g} \sim \ell_{c}$. The losses can be neglected if

$$
R \gg \frac{R_{\mathrm{opt}}}{\Gamma^{3}},
$$

where the optimal size is the function of critical energy $\varepsilon_{2}$, which is defined as if the magnetic field were uniform.

In order to be in agreement with the observed diffuse gamma-ray background, a typical source of the highestenergy CRs must satisfy the condition $R \gtrsim 0.2 R_{\text {opt }}$ [2], so that the synchrotron losses during diffusion do not lead to any further resrictions. The aforementioned statistical arguments are not applicable to individual abberrant accelerators, for which Eq. (20) or Eq. (21) should be considered as an additional limit.

The critical energies in the case of acceleration by a shear flow are

$$
\varepsilon_{1}=\varepsilon_{2}=\Gamma e B R
$$

for uniform magnetic field and

$$
\varepsilon_{1}=\Gamma e B \sqrt{R \ell_{c}} \quad \text { and } \quad \varepsilon_{2}=\Gamma^{\frac{3}{2}} e B \sqrt{R \ell_{c}}
$$

for chaotic field. It is interesting to note, that in the latter case $\varepsilon_{2}>\varepsilon_{1}$, i.e., the maximum energy achievable in 
the converter mechanism appears to be $\Gamma^{1 / 2}$ times larger than that in the standard mechanism. However, a particle can only attain this energy if it enters acceleration cycle with the energy $\varepsilon \sim \varepsilon_{2} / \Gamma^{2}$. A particle, entering the cycle with larger energy, will end up with smaller energy, in contrast to the other three cases.

Of course, every acceleration mechanism must obey the fundamental restrictions and constraints of classical electrodynamics 2]. Among them is the Hillas criterion [18, 19] generalized for relativistic bulk flows, which is simply equivalent to the condition $\varepsilon<\varepsilon_{1}$, where $\varepsilon_{1}$ is the first critical energy from Eqs. (14) and (22). This is not a chance coincidence, but rather the consequence of the implied - uniform - magnetic field configuration. In a turbulent magnetic field, the maximum attainable energy is always lower than the fundamental limits, but the converter mechanism is less affected by the turbulence. Indeed, the magnetic field should be considered chaotic if its turbulence scale is less than the gyroradius of a particle at the beginning of the acceleration cycle. It means $\ell_{c}<r_{g}\left(\varepsilon_{1}\right)$ for the standard mechanism, but only $\ell_{c}<r_{g}\left(\varepsilon_{2} / \Gamma^{2}\right)$ for the converter mechanism. Thus, a particle accelerated in the standard way feels turbulence starting from larger scale, and a situation is possible, where the magnetic field should be treated as chaotic in the standard mechanism while being essentially uniform for the converter mechanism. The inequality $\varepsilon_{2}>\varepsilon_{1}$ from Eq. 231) is, in fact, the consequence of slower decrease of critical energy with decreasing $\ell_{c}$ for the converter mechanism.

So far, the analysis was limited to the case of negligible synchrotron losses and $\tau \ll 1$. The larger is the probability of conversion, the easier is acceleration in the converter mechanism. But if $\tau$ approaches unity, then the critical energies decrease: they are still given by Eqs. (14), (16), (22), and (23), but the value of $R$ should be replaced by the (energy-dependent) mean free path of the accelerated particle. Analogously, the synchrotron losses also decrease the critical energies; in this case $R$ should be replaced by the radiation length.

In both cases the energy share of the accompanying radiation is non-negligible or even dominant, although the status of conversion losses is qualitatively different for the proton and electron cycles (see Sect. V).

\section{RESULTING PARTICLE DISTRIBUTION}

Let us consider a monoenergetic $\left(\varepsilon=\varepsilon_{0}\right)$ beam of particles in the neutral state, escaping from the relativistic flow. If the probability of conversion per unit length, $\lambda$, is constant (i.e., we neglect the exponential decrease in the number of particles, assuming $\tau \ll 1$ ), then the distribution of charged particles over initial displacement $d N / d \ell_{0}=\lambda$ translates into

$$
\frac{d N}{d \varepsilon} \propto \frac{d N}{d \ell_{0}} \frac{d \ell_{0}}{d g} \propto \lambda\left(\theta \frac{d \theta}{d \ell_{0}}\right)^{-1}
$$

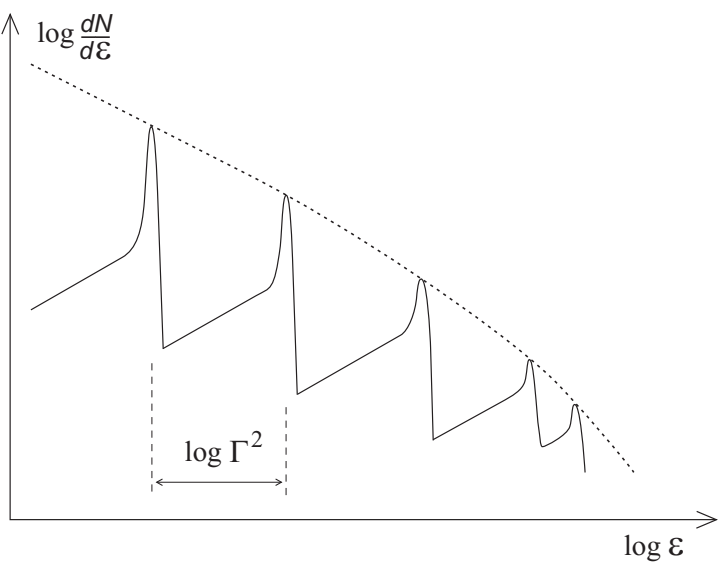

FIG. 3: The particle distribution (solid line), resulting from a monoenergetic injection. The dips in the distribution preserve themselves until the width of the injection spectrum is larger than $\log \Gamma^{2}$ in logarithmic units.

where the particle's energy is simply proportional to the energy gain, $\varepsilon=g \varepsilon_{0}$. It is a power-law distribution, but all the particles with displacement larger than the applicability limits of Eqs. (13), (15), (18), and (19) have the constant energy gain of $\sim \Gamma^{2}$, so that in general a delta-function is added to the resulting particle distribution at its high-energy end. In their power-law parts, the distributions emerging in uniform and chaotic field, respectively, are

$$
\frac{d N}{d \varepsilon} \propto \varepsilon^{1 / 2} \quad \text { and } \quad \frac{d N}{d \varepsilon} \propto \varepsilon
$$

for acceleration at the shock front, and

$$
\frac{d N}{d \varepsilon}=\text { const } \quad \text { and } \quad \frac{d N}{d \varepsilon} \propto \varepsilon^{1 / 2}
$$

for acceleration at the boundary of shear flow. These spectra are extremely hard. In practice, they can be considered delta-functions, so that the resulting distribution is defined mainly by the spectrum of injected particles.

After a few cycles, a seesaw-shaped spectrum (Fig. 3) is formed from the initial monoenergetic distribution. The number of particles emerging from a cycle, $N^{\prime}$, is related to the number of particles entering it as $N^{\prime}=k N$, where

$$
k=p_{\mathrm{esc}} \prod_{i} p_{c n}^{(i)}
$$

is the overall probability of conversion, which normally occurs twice per cycle (points 1 and 2 in Fig. 2), multiplied by the probability of escape from the relativistic flow after the first conversion $p_{\text {esc }}$, which is the relative number of particles moving upstream with respect to the shock front or the shear flow boundary. Assuming that the particle distribution is isotropic just before the conversion into neutral state, one obtains $p_{\text {esc }}=1 / 3$ for a strong relativistic shock, where the shocked fluid 
moves at the speed $c / 3$ away from the shock plane, and $p_{\text {esc }}=1 / 2$ for a shear flow, where the fluid velocity is parallel to the boundary. Within one acceleration cycle, the probabilities $p_{c n}^{(1)}$ and $p_{c n}^{(2)}$ are of the same order, except for the case where $p_{c n}^{(2)}$ is limited by Eq. (8).

There is a simple case, where $k$ remains constant in the course of acceleration. This happens in both electron and proton cycles if the spectrum of target photons is a power-law with spectral index $q=0$ or (for the proton cycle only) in the case of a narrow-band target-photon field. Then the envelope of the particle distribution shown in Fig. 3 is a power-law $d N / d \varepsilon \propto \varepsilon^{-\alpha}$, and the index $\alpha$ can be obtained in the following way.

Since the particle energies are related as $\varepsilon^{\prime}=g \varepsilon$, we find that

$$
\left(\frac{d N}{d \varepsilon}\right)^{\prime}=\frac{k}{g} \frac{d N}{d \varepsilon}
$$

where primed is the distribution emerging from the cycle. Taking logarithm of both parts, we obtain

$$
-\alpha \ln g=\ln \left(\frac{k}{g}\right) \quad \Rightarrow \quad \alpha=1-\frac{\ln k}{\ln g} .
$$

If the probability of passing through the acceleration cycle $k$ is larger than the inverse energy gain $1 / g$, then the spectral index is $\alpha<2$, i.e., most of the energy content is at the high-energy end of the distribution. Both AGNs and GRBs can satisfy this requirement (see Eqs. 5] 6] and (7) and, therefore, can be efficient producers of the highest-energy cosmic rays.

In the energy range, where the efficiency condition for the converter mechanism is satisfied, this mechanism is the dominant source of accelerated particles - just because it provides a spectrum harder than the one resulting from the standard mechanism. At the same time, there is no actual threshold in the conversion probability: the converter mechanism can function even at $p_{c n} \rightarrow 0$, but the number of the accelerated particles in this case is extremely depleted at high energies. Quantitative studies of the emerging CR spectrum must take into account the dependence of the conversion probability on the energy of accelerated particles. Hence, the precise solution can be obtained only in the self-consistent approach, which includes the effect of the accompanying emission, discussed in the following section.

\section{THE ACCOMPANYING EMISSION}

Acceleration of particles via the converter mechanism is inevitably accompanied by gamma-ray and - in the case of nucleon conversion - by neutrino emission. The conversion losses in the electron cycle, which are the result of non-optimal inverse Compton scatterings, do not lead to irreversible energy drain. Instead, all the energy remains in photons or electrons (positrons) and, as they both can in principle participate in the acceleration cycle, may be used to inject new particles for acceleration. Thus, even in the case $\tau \gg 1$ the influence of the conversion losses is reduced to the decrease of maximum attainable energy and the increase in the level of synchrotron emission by rising the number of particles involved in the acceleration.

On the contrary, the conversion losses in the proton cycle act as a true energy sink: the energy spent for the pion production never comes back to the accelerated nucleons. Neutrinos, $e^{-} e^{+}$-pairs and gamma-rays, which are the decay products of pions, are copiously produced as byproducts of the proton acceleration cycle. Neutrinos carry away about one-half of the energy of accelerated nucleons and, because they freely escape from the acceleration site, their spectrum copies that of the nucleons, but scaled down in energies by about an order of magnitude. The gamma-rays and pairs, which carry approximately the same energy as neutrinos, are reprocessed through the electromagnetic cascade: the photons are absorbed in two-photon pair production process, electrons and positrons cool in the magnetic field, producing synchrotron radiation, i.e., another generation of photons, etc.

The hard spectrum of primary electrons and positrons means that they form a standard cooling distribution, $d N_{e} / d \gamma_{e} \propto \gamma_{e}^{-2}$, and their synchrotron spectrum is a power-law with (photon) index $-3 / 2$. The synchrotron photons spawn another generation of pairs, which cool to form a distribution with index $-5 / 2$. The cascade comprises several steps like this, so that the photon spectrum eventually converges to the power-law with index -2 . However, at low energies the acceleration site is transparent for the photons, and therefore pairs are not injected below some energy, which leads to a break in the spectrum; below the break the photon spectrum preserves its original spectral index $-3 / 2$. The location of the break can be found in a self-consistent way:

$$
\varepsilon_{\mathrm{br}} \simeq 0.5 \frac{\hbar e B}{\Gamma m_{e} c}\left(\frac{\varepsilon_{\mathrm{tr}}}{2 m_{e} c^{2}}\right)^{2},
$$

where $\hbar$ is the Planck constant. The threshold photon energy $\varepsilon_{\text {tr }}$ is defined so that the optical depth for twophoton pair production,

$$
\tau_{\gamma \gamma}\left(\varepsilon_{\mathrm{tr}}\right)=\frac{\sigma_{\gamma \gamma}}{\sigma_{\pi}} \tau_{\pi} \frac{\varepsilon_{\mathrm{br}} \varepsilon_{\mathrm{tr}}}{2 \Gamma^{2} m_{e}^{2} c^{4}}\left(\frac{\varepsilon_{*}}{\varepsilon_{\mathrm{br}}}\right)^{1 / 2},
$$

is equal to unity, where $\sigma_{\gamma \gamma}$ is the cross-section of twophoton pair production and $\tau_{\pi}$ the optical depth for photopionic reactions at photon energy $\varepsilon_{*}$. Straightforward calculations yield

$$
\varepsilon_{\mathrm{br}} \simeq 0.5 \frac{\sigma_{\pi}}{\sigma_{\gamma \gamma} \tau_{\pi}} \sqrt{\frac{\hbar e B}{m_{e} c} \frac{\Gamma \varepsilon_{N} m_{e}^{2} c^{2}}{\Delta_{p} m_{N}}} .
$$

Substituting $B=10^{3} \mathrm{G}, \varepsilon_{N}=10^{19} \mathrm{eV}$ (the energy of accelerated nucleons), $\Gamma=1000$, and $\tau_{\pi}=10^{-2}$, - 
parameters, reasonable for GRBs, - we obtain the break energy $\varepsilon_{b r} \sim 100 \mathrm{keV}$, which is similar to the really observed in GRBs spectral features. Within this picture, however, there is no simple way to explain the observed relative stability of $\varepsilon_{\text {br }}$ whereas the parameters entering Eq. (32), especially $\tau_{\pi}$, may vary by orders of magnitude.

Unlike the conversion losses, the synchrotron emission is not tightly related to the acceleration process as such. It may have a negligible effect, especially for protons, but also may be the main energy loss channel. The detailed analysis of the properties of accompanying synchrotron radiation is beyond the scope of this paper, but we point out two distinctive features.

First, the maximum energy of synchrotron photons for the converter mechanism is $\Gamma^{2}$ times larger than for the standard one. The existence of such an energy limit is easy to see for the standard mechanism (following the arguments of [20]). The acceleration cycle in this case lasts $\sim r_{g} / c$ and the energy increment is $\sim \varepsilon$, which gives the acceleration rate $\dot{\varepsilon} \sim \varepsilon c / r_{g}$. The maximum admissible rate of synchrotron losses is just the same, so that the particle's energy is limited by the following inequality:

$$
\frac{4}{9}\left(\frac{e^{2}}{m c^{2}}\right)^{2} B^{2}\left(\frac{\varepsilon}{m c^{2}}\right)^{2}<\frac{\varepsilon}{r_{g}}
$$

Then, simple calculation yields the maximum energy of synchrotron photons (ignoring relativistic dipole radiation caused by small-scale inhomogeneities of magnetic field):

$$
\varepsilon_{\mathrm{sy}}^{(\max )} \sim 0.5 \frac{\hbar e B}{m c}\left(\frac{\varepsilon}{m c^{2}}\right)^{2} \sim \frac{\hbar c}{e^{2}} m c^{2} \simeq 137 m c^{2} .
$$

Doppler boosting gives additional factor $\Gamma$, and in a turbulent magnetic field with the spatial scale of the turbulence less than $\ell_{c}^{\text {cr }}=m^{2} c^{4} / \sqrt{e^{5} B^{3}}$ another factor $\left(\ell_{c} / \ell_{c}^{\mathrm{cr}}\right)^{2 / 3}$ applies.

The same reasoning is valid for the converter mechanism, in which the cycle duration is $\geq r_{g} / c$ and the energy increment is $\leq \Gamma^{2} \varepsilon$. Consequently, the analog of Eq. (33) gives $\Gamma^{2}$ times larger limit for the energy of accelerated particle, which translates into factor $\Gamma^{4}$ in the expression for the energy of synchrotron photons. When the accelerated particle enters the relativistic flow being close to the limiting energy, the synchrotron emission is so efficient, that the particle loses almost all its energy before it is deflected by an angle $\sim 1 / \Gamma$. Thus, the resulting synchrotron emission is beamed backwards in the flow comoving frame. In the laboratory frame it appears redshifted by the factor $\Gamma$, in contrast to the standard mechanism, in which the synchrotron emission is blueshifted in the laboratory frame by the same factor $\Gamma$. Thus, for an observer resting in the laboratory frame, the maximum energy of synchrotron photons accompanying the converter-acceleration is by the factor of $\sim \Gamma^{2}$ larger (recall the difference of $\sim \Gamma^{4}$ in the comoving frame), than in the standard mechanism. Moreover, this highest-energy synchrotron radiation is quasi-isotropic in the laboratory frame, which is another distinctive feature of the converter mechanism. The latter phenomenon has a nature similar to the effect of beam-pattern broadening for the inverse Compton radiation of electrons in front of relativistic shock [21]. Generally speaking, the converter mechanism makes neutral beams of all kinds (photon, neutrino and neutron beams) broader than $1 / \Gamma$, so that they can be seen even if the jet that produced them is not pointing towards the observer.

The accompanying electromagnetic emission of any origin can itself provide photons for the conversion reactions, which may give rise to radiative instabilities analogous the instability driven by $p+\gamma \rightarrow p+e^{-}+e^{+}$process, discussed in [22].

\section{DISCUSSION}

The key parameter of the converter mechanism - the probability of conversion - varies from one cycle to another or even within cycles. It increases in the course of acceleration (because a more energetic accelerated particle interacts with less energetic and more abundant target particles) and with increase of the angle between the particle's trajectory and the velocity of the flow. The first effect is negligible for nucleon-nucleon collisions, while the second is partially or even completely compensated by smaller distance travelled at large angles. There is, however, a general trend of increase of the probability of conversion towards higher energies of accelerated particles; this may even block further acceleration if $p_{\mathrm{cn}}$ approaches unity. Nevertheless, with the expansion of the flow the density of target particles drops, leading to the decrease of the probability of conversion, and the acceleration resumes.

It means, that the converter mechanism is capable of self-tuning. The only thing which is required for this mechanism to be efficient is that the probability of passing through the acceleration cycle $k$ is larger than $1 / \Gamma^{2}$ somewhere along the flow. It does not matter how large is the probability: in the case $k \rightarrow 1$ the particles are preaccelerated in the region of high optical thickness and then are further accelerated when (or where) the flow becomes optically thin for them. Of course, the question how much energy is wasted for conversion losses during this intermediate phase is open.

On the other hand, the condition $k>1 / \Gamma^{2}$ places some restrictions on the sources where the proton cycle is readily realizable starting from thermal protons. If a source is known to be bright at hard gamma-rays, one can conclude that the source's compactness, calculated for the corresponding target photons, is low, so that the probability of proton-to-neutron conversion is $k \lesssim \sigma_{\pi} / \sigma_{\gamma} \simeq 3 \times 10^{-3}$. Thus, the source should have $\Gamma \gtrsim 20-$ a condition, satisfied by GRBs and many AGNs. Moreover, this condition is not obligatory for the sources, where the converter mechanism starts from preacceler- 
ated high-energy protons, which require only low-energy target photons.

We expect that the converter mechanism never operates alone and the standard Fermi-type mechanism competes with it. Because the particles spend some time in the neutral state, the converter mechanism has smaller acceleration rate at low energies. But, close to the limiting energy $\left(\varepsilon_{1}\right)$, the durations of acceleration cycle in both mechanisms approach the same value $R / c$, so that the average acceleration rates are roughly equal. Except for the case of acceleration in a shear flow with chaotic ambient magnetic field, the mechanisms have just the same absolute, i.e., ignoring radiative losses, energy limit for the accelerated particles. However, in the converter mechanism, this energy is attained in much fewer steps, with potentially much more particles survived. Moreover, the particles - when converted into neutral state - can escape from regions located deep inside the relativistic flow, which further reduces irreversible particle losses in the downstream. So, at the highest energy part of the distribution almost all particles are produced by the converter mechanism, regardless of its performance at low energies.

Now let us consider the phase, when produced CRs leave the accelerator. Within the framework of the converter mechanism, the particles can escape in the form of neutral beam - an easy way, which causes no problem and requires nothing but a sufficiently high conversion probability. In the standard scheme, the escaping particles are charged and inevitably must form an expanding turbulent outflow. This causes considerable adiabatic losses, which can hardly be controlled. Another advantage of the converter mechanism is its greater tolerance for non-uniform magnetic fields, as discussed in Sect. III. Since the magnetic field turbulence has strictly negative effect on the maximum attainable energy, the converter acceleration mechanism may have a larger cut-off energy.

Unlike the standard mechanism, the converter mechanism in many cases does not need any special particle injection. By definition of acceleration cycle, even the particles resting either in the laboratory or in the flow frame serve as injection as soon as they are converted into neutral state. Sometimes however, e.g., in AGNs, the probability of conversion is too small at low energies to provide sufficient injection. Then, the converter mechanism comes to depend on the standard mechanism, which produces preaccelerated particles (at energies $\gtrsim 10^{15} \mathrm{eV}$ in the case of AGNs).

The photopionic processes in AGNs and GRBs leading to copious production of neutral particles have been extensively discussed in the literature (e.g., 14, 23 ), in particular in the context of predictions of fluxes of high energy neutrinos from these objects. The main reasoning was that these objects are postulated to be efficient sources of high-energy CRs and they are surrounded by dense photon fields. Therefore, nucleon-photon interactions may lead to detectable neutrino fluxes. Our point of view is essentially different: if one wants to accelerate
CRs in these objects to highest energies, then the converter mechanism is likely the most efficient way to do this, and the neutrino emission appears as a natural and unavoidable byproduct of this mechanism.

Further questions, absent in the test particle problem, arise in the self-consistent approach. In particular, the converter mechanism strongly alters hydrodynamics of relativistic shocks or shear flows. We have shown that they can accelerate nucleons with the efficiency of energy transfer approaching $100 \%$. At such a high efficiency, almost all the available energy is transferred to the end of the particle distribution, and hence the ultra-energetic particles contribute most of the inertia of the relativistic flow (shocked gas). Under such circumstances, the idea of a shock as a discontinuity becomes meaningless, since the gyroradius of dynamically most important particles is comparable to the size of the system.

This limits applicability of the test particle approach, since it is only valid for the particles whose gyroradius is larger than the width of the shock or the shear flow boundary. The similar problem exists also in the standard mechanism, which is, moreover, more sensitive to it. Indeed, in the converter mechanism an accelerated particle may cross the shock or the shear flow boundary while being in the neutral state, hence relaxing the requirements to their sharpness.

The absence of a true shock is an obstacle for the electron cycle, which would otherwise be a very efficient way of direct acceleration of electrons. When the mean free paths of photons and pairs are small compared to the spatial scale of velocity gradient, the direct acceleration of electrons probably cannot rival the efficiency of production of secondary pairs via nucleon acceleration. One of the implications is that at least in some, particularly bright, GRBs the main source of the observed gamma-ray radiation is the pion-induced cascade.

Energetic neutrons (or photons) escape from the relativistic flow and their decay or interaction products can disturb the ambient medium to the extent that it starts to move with an ultrarelativistic speed. An apparent consequence of this is the distortion of beam pattern of the source, while the influence on the limiting energy of the accelerated particles needs further investigation. On one hand, the decrease in the Lorentz factor contrast lowers the limiting energy. On the other hand, enhancement of the ambient magnetic field, either via compression in the shock or by means of various instabilities triggered by neutron decay products in the ambient plasma, has the opposite effect.

\section{SUMMARY}

In this paper we suggest and analyze a new acceleration mechanism, which operates via continuous conversion of accelerated particles from charged into neutral state and back. The proposed converter mechanism is efficient for acceleration of both protons and electrons (positrons). It 
is capable of producing the highest-energy $\left(\gtrsim 10^{20} \mathrm{eV}\right)$ cosmic rays in either GRB or AGN environments. With a much lower energy limit, the mechanism can possibly operate in microquasars as well. In the regions of high optical thickness, the converter mechanism is an efficient means of transferring the kinetic energy of bulk relativistic flow to the accompanying radiation, which could explain, for example, the origin of GRB emission. Some peculiarities of the accompanying emission can be a telltale sign of the converter mechanism. For example, the production of the highest-energy CRs should be linked with the powerful neutrino emission at a level at least comparable to the power in CRs.

Despite a certain similarity to the standard (diffusive) acceleration mechanism, the converter mechanism violates some of its inherent relations. For example, the maximum particle energy attainable in the converter mechanism is $\Gamma^{1 / 2}$ times larger than in the standard one, provided acceleration occurs in a shear flow with chaotic ambient magnetic field. Also, the maximum energy of synchrotron photons appears to be $\Gamma^{2}$ times larger.
Generally speaking, a beam pattern wider than $1 / \Gamma$ is characteristic for any type of accompanying emission (synchrotron radiation at highest energies, neutrino emission or photons from pion decay), as well as for the escaping neutrons. This distinctive feature of the converter mechanism opens an interesting possibility for observation of the off-axis blazars and GRBs.

\section{Acknowledgments}

We thank John G. Kirk and Michal Ostrowski for useful discussions and the referee for his suggestions. This work was partially supported by the grants 02-02-16236 of the Russian Foundation for Basic Research and 00-1596674 of the Council for Support of the Leading Scientific Schools, and by programs "Astronomia" of the Presidium of the Russian Academy of Science and of the Ministry of Industry, Science and Technology.
[1] M. Nagano and A. A. Watson, Rev. Mod. Phys. 72, 689 (2000).

[2] F.A. Aharonian, A.A. Belyanin, E.V. Derishev, V.V. Kocharovsky, Vl.V. Kocharovsky, Phys. Rev. D 66, id. 023005 (2002).

[3] J.E. Gunn and J.P. Ostriker, Phys. Rev. Lett. 22, 728 (1969).

[4] M.A. Malkov and L.O'C. Drury, Rep. Prog. Phys. 64(4), 429 (2001).

[5] E.G. Berezhko, JETP Lett. 33(8), 399 (1981).

[6] M. Ostrowski, A\&A 335, 134 (1998).

[7] A. Levinson, D. Eichler, Astrophys. J. 418, 386 (1993).

[8] M. Vietri, Astrophys. J. 453, 883 (1995).

[9] E. Waxman, Phys. Rev. Lett. 75, 386 (1995).

[10] J. Bednarz and M. Ostrowski, Phys. Rev. Lett. 80, 3911 (1998).

[11] A. Achterberg, Y.A. Gallant, J.G. Kirk, and A.W. Guthmann, Mon. Not. RAS 328, 393 (2001).

[12] E.V. Derishev and F.A. Aharonian "Hadron acceleration in Gamma-Ray Bursts and its implications for the burst dynamics", Proceedings of the 3rd Workshop "GammaRay Burst in the Afterglow Era", 17-20 September 2002, Rome, Italy, (2003, in press).
[13] B. Stern, astro-ph/0301384 (2003).

[14] J.P. Rachen and P. Mészáros, Phys. Rev. D 58, id. 123005 (1998).

[15] P. Mészáros and M.J. Rees, ApJ 541, L5 (2000).

[16] C.M. Urry and P. Padovani, PASP 107, 803 (1995).

[17] P. Mészáros, Annu. Rev. Astron. Astrophys. 40, 137 (2002).

[18] K. Greisen, in Proceedings of the 9th International Cosmic Ray Conference (Institute of Physics, London, 1965), Vol. 2, p. 609.

[19] A.M. Hillas, Annu. Rev. Astron. Astrophys. 22, 425 (1984).

[20] P.W. Guilbert, A.C. Fabian, M.J. Rees, MNRAS 205, 593 (1983).

[21] E.V. Derishev, V.V. Kocharovsky, Vl.V. Kocharovsky, Proc. of the "High Energy Gamma-Ray Astronomy" Symposium, 26-30 June, 2000, Heidelberg, Germany, eds. F.A. Aharonian and H.J. Voelk. AIP Proceedings, v.558, p.405 (2001).

[22] J.G. Kirk and A. Mastichiadis, Nature 360, 135 (1992).

[23] A.M. Atoyan and C.D. Dermer, ApJ 586, 79 (2003). 